\title{
Editorial
}

\section{Nanofiber Manufacture, Properties, and Applications 2013}

\author{
Tong Lin, ${ }^{1}$ Gajanan S. Bhat, ${ }^{2}$ and Raghavendra Hegde ${ }^{3}$ \\ ${ }^{1}$ Australian Future Fibres Research and Innovation Centre, Institute for Frontier Materials, Deakin University, 75 Pigdons Road, \\ Waurn Ponds, VIC 3216, Australia \\ ${ }^{2}$ Nonwovens Research Laboratory, The University of Tennessee, 1321 White Avenue, Knoxville, TN 37996, USA \\ ${ }^{3}$ Domtar Personal Care, 2332 US Highway 42 South, Delaware, OH 43015, USA \\ Correspondence should be addressed to Tong Lin; tong.lin@deakin.edu.au
}

Received 12 January 2014; Accepted 12 January 2014; Published 18 February 2014

Copyright (C) 2014 Tong Lin et al. This is an open access article distributed under the Creative Commons Attribution License, which permits unrestricted use, distribution, and reproduction in any medium, provided the original work is properly cited.

Nanofibers possess versatile properties and wide applications. Although they can be prepared by several different methods, electrospinning is distinct from other nanofiber-making techniques in versatility to process different polymers, ability to control fiber diameter, morphology, orientation, component and fibrous structure, and potential for large-scale production. The ease of electrospinning nanofibers on small scales, mainly using a needle-based electrospinning setup, in laboratories has considerably facilitated researchers' finding of the unique properties and applications of electrospun nanofibers.

This special issue highlights the functional applications of electrospun nanofibers in gas sensor, drug delivery, and electronics fields. A planar-type methane sensor was prepared from a doped electrospun $\mathrm{SnO}_{2}$ nanofiber. The sensor was reported to have good stability, prominent reproducibility, and excellent selectivity. In comparison with $\mathrm{SnO}_{2}$ nanospheres, which were used conventionally for making methane sensors, the nanofibers had higher gas response and saturated detection concentration, as well as quicker response-recovery time.

Controlling drug release has many applications in the biomedical field. In one of the papers on drug delivery nanofibers, drug was loaded in polymer/silica hybrid nanofibers. The presence of the silica remarkably enhanced drug loading efficiency, nanofiber hydrophilicity, and mechanical properties. Assessed by in vitro cell tests, the hybrid nanofibers showed good biocompatibility for guided bone regeneration. Another paper reports drug loaded nanofibers which were electrospun directly onto a polymeric backing film. The optimized nanofiber/film matrix system showed enhancement in drug dissolution rate, useful for rapid mucosal drug delivery.

Two papers on electronics applications of electrospun nanofibers were included in this special issue. One paper reports on the $p-n$ junction and rectifying property of a layered fibrous mat consisting of inorganic nanofibers and conducting polymer nanofibers. The fibrous mat had an obvious diode-rectifying characteristic, and the thickness of the nanofiber layers considerably influenced the device resistance and rectifying performance. Such nanofibrous diode rectifier may find applications in sensors, energy harvest, and electronic textiles. Another paper is on electrode connection solder. Short circuit often occurs at fine pitch interconnections of electronic devices. To solve this problem, solder balls were incorporated into polyvinylidenefluoride nanofibers through electrospinning. The nanofiber solder showed considerable improvement in solder capture rate and $100 \%$ insulation between electrodes. It also improved contact conductivity, current handling ability, and reliability.

The special issue also collects fundamental research on electrospinning. Electric current between electrospinning electrodes originates from the movement of charge carriers through the spinning space. It was reported that the majority of charge carriers were formed by ionization of the air close to the metallic needle nozzle or the polymer jet. The addition of salt to polymer solution increased the carrier concentration. However, the conductivity of polymer jets did not significantly affect the current since the jets did not link the electrodes. 
Apart from electrospun nanofibers, a paper on multiwalled carbon nanotube (MWCNT)/polyamide 66 composites is also included in this special issue. MWCNTs with surfaces modified separately with acid and amine groups were used as fillers. The amine-modified MWCNTs showed better dispersion in the polymer matrix than pristine- and acidmodified MWCNTs. They induced heterogeneous nucleation of PA66 and significantly improved the composite hardness and elastic modulus.

As guest editors of this special issue, we are pleased to see this progress. We hope that this special issue will contribute to the wide use of nanofibers and that the papers collected in this special issue are well received by the reader.

\section{Acknowledgments}

The editors gratefully thank the authors for their contributions to this special issue and the reviewers for their constructive comments and dedication.

Tong Lin

Gajanan S. Bhat

Raghavendra Hegde 

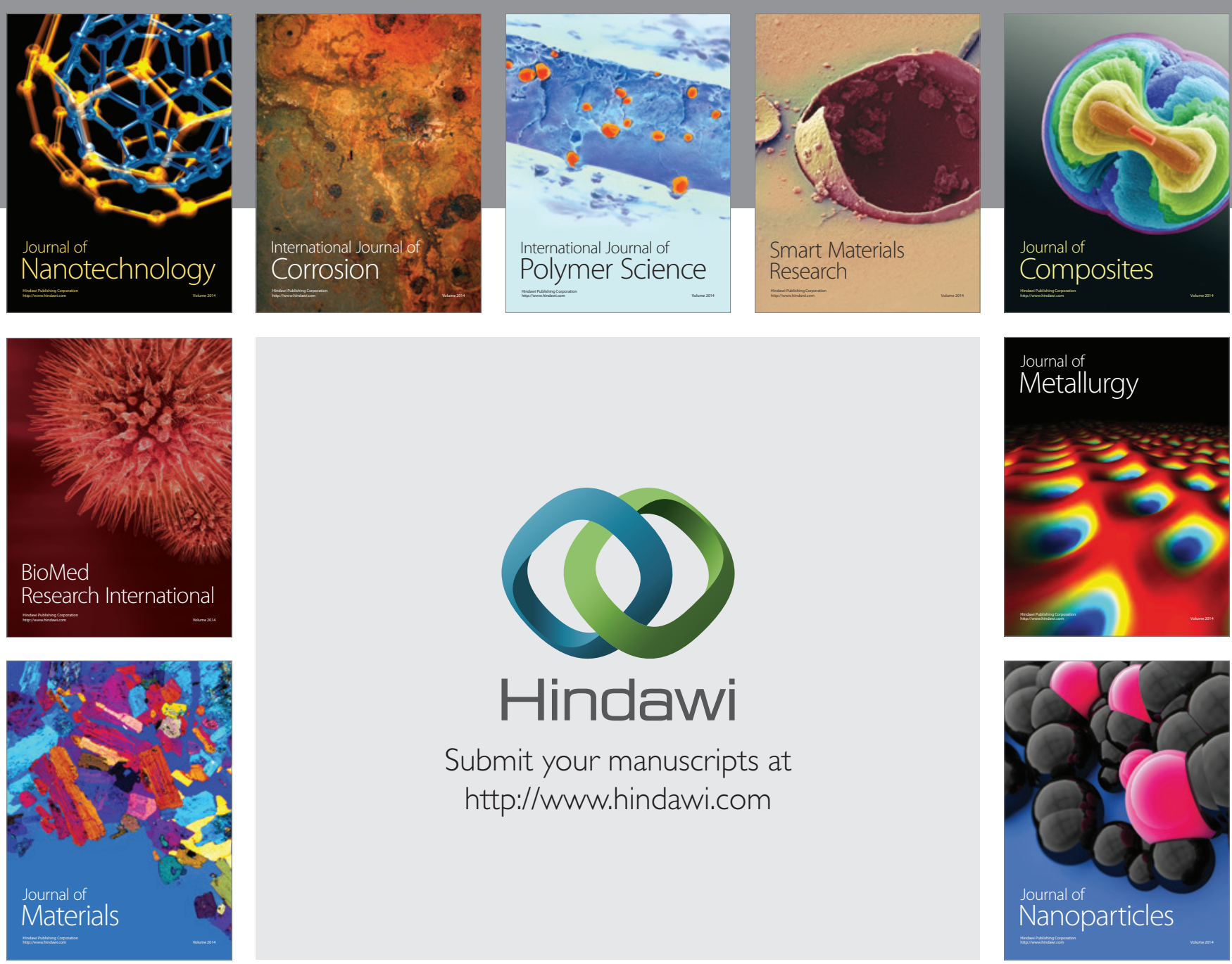

Submit your manuscripts at http://www.hindawi.com
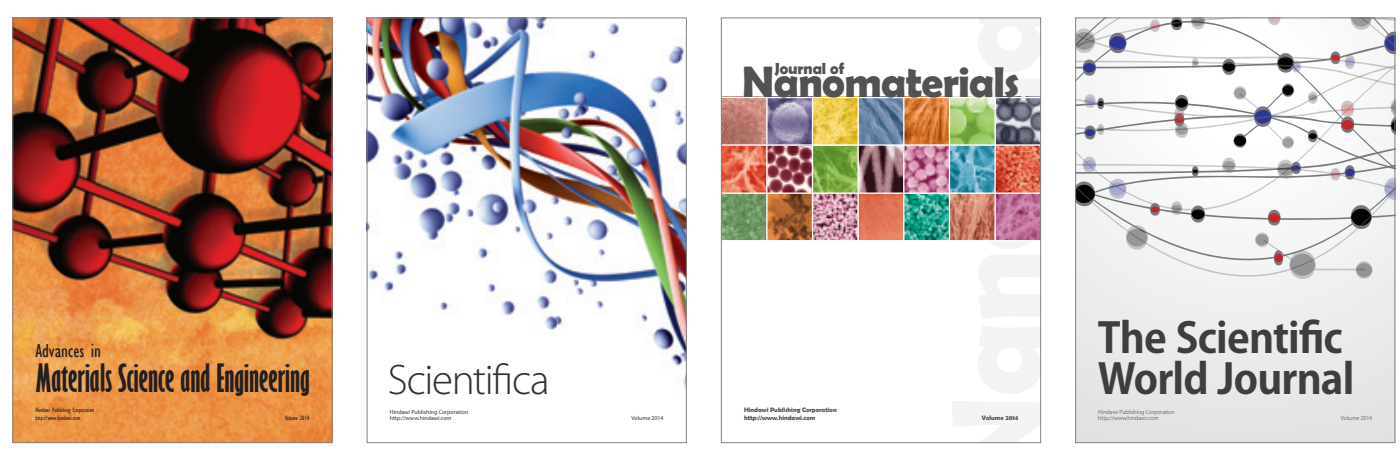

\section{The Scientific World Journal}
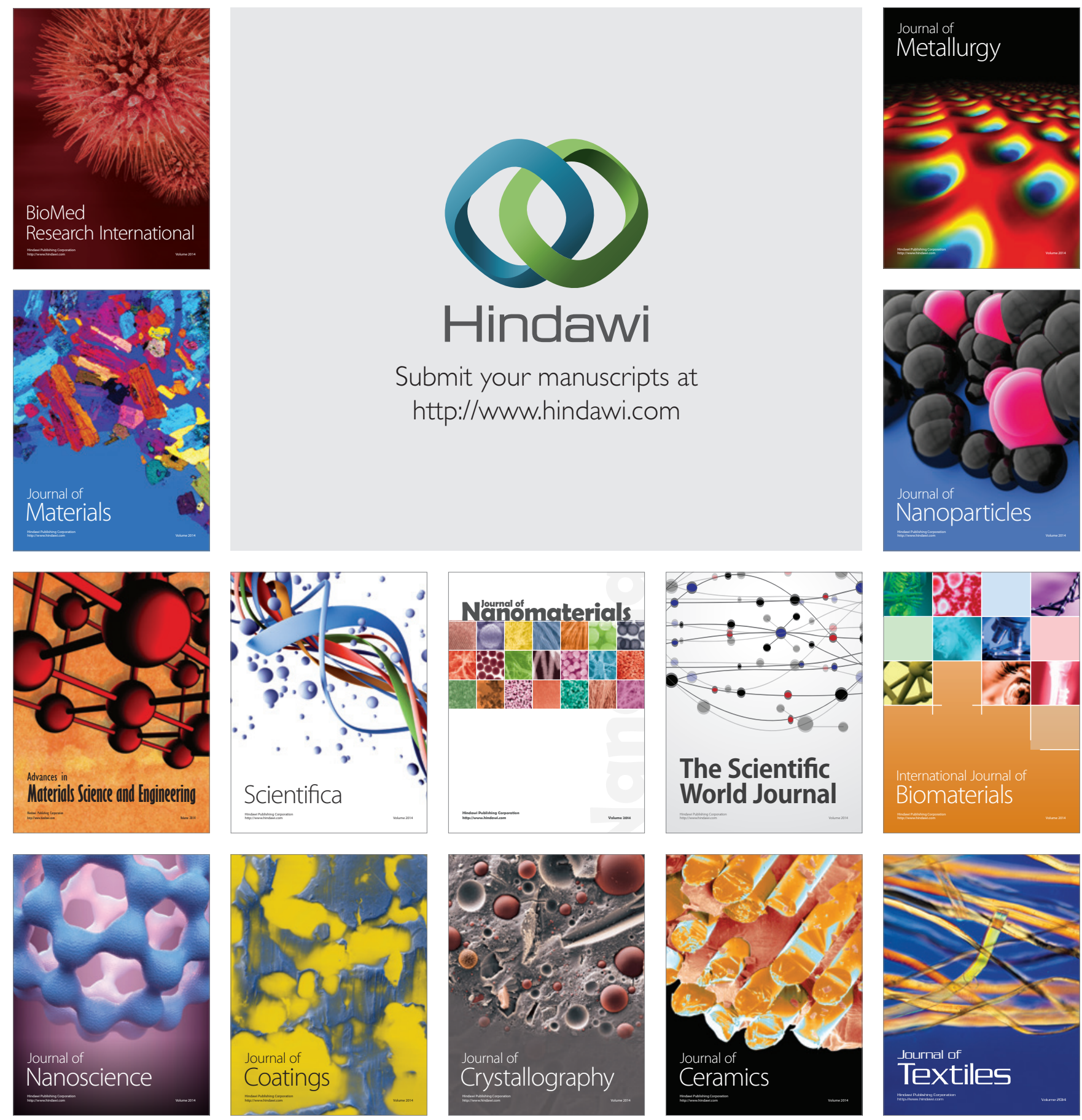